\title{
Educação permanente para avaliação em saúde: uma aposta na arte do encontro
}

Permanent education for health evaluation: a bet on the art of meeting

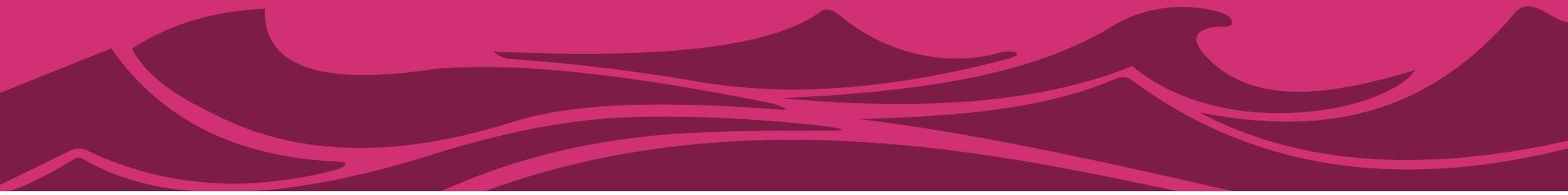

\section{Marcela Silva da Cunha}

Mestre em Saúde Pública. Subárea Planejamento e Gestão de Sistemas e Serviços de Saúde pela Escola Nacional de Saúde Publica (ENSP/FIOCRUZ). Especialista em Avaliação de Serviços em Saúde com ênfase em Educação Permanente pela Universidade Federal de Ciências da Saúde de Porto Alegre (UFCSPA), Especialista em Formação Integrada Multiprofissional em Educação Permanente em Saúde pela Universidade Federal do Rio Grande do Sul (UFRGS) e graduada em Psicologia pela Universidade do Estado do Rio de Janeiro (UERJ). Tem experiência em docência, pesquisa e gestão nas área de Saúde Coletiva, Saúde da Família / Atenção Básica e Planejamento em Saúde.

E-mail: mcscelcunha@gmail.com

\section{Eric Campos Alvarenga}

Possui graduação em Psicologia pela Universidade Federal do Pará (2009). Mestrado em Psicologia pela UFPA (2009) e, atualmente, é doutorando do Programa de Pós-graduação em Psicologia da UFPA. Trabalha como supervisor master do Programa Nacional de Melhoria do Acesso e da Qualidade da Atenção Básica (PMAQ), projeto do Ministério da Saúde e é coordenador do Grupo de Estudos em Saúde na Amazônia. Tem atuado lecionando e fazendo pesquisas nas áreas de Psicologia do Trabalho, Saúde do Trabalhador e Saúde Coletiva.

E-mail:ericsemk@gmail.com

\section{Resumo}

O objetivo deste artigo é apresentar a vivência de tutoria no Curso de Especialização em Avaliação de Serviços em Saúde, discutindo a Educação Permanente e sua relação com a Avaliação. O curso teve início em conjunto com a avaliação externa do 2o Ciclo do Programa Nacional de Melhoria do Acesso e da Qualidade (PMAQ) a partir de janeiro de 2014 no estado do Pará. Os tutores e tutoras da especialização também coordenavam equipes de profissionais que coletavam dados referentes às equipes de saúde da família do estado. Foram criados espaços para discutir aspectos decorrentes do processo de avaliação e estranhamentos provocados pelo cotidiano de trabalho. A organização do trabalho era, desse modo, compartilhada e discutida pelas equipes de coletadores. O curso de especialização mostrou-se rico em trocas de experiências com alunos de diversos estados, porém com poucos espaços de participação ativa de discentes na construção do curso. Uma cultura de avaliação de serviços de saúde está sendo germinada 
pelo PMAQ por meio de mudanças de comportamento impulsionadas que podem viabilizar melhores resultados na saúde da população. Desta forma, a experiência de avaliação precisa ser entendida como um processo social e político de transformação.

Palavras-chave: Educação Permanente; Avaliação em Saúde; Atenção Básica.

\section{Abstract}

The aim of this article is to present the experience of tutoring in the Specialization Course in Health Services Evaluation, discussing the Permanent Education and its relation with the Evaluation. The course began in conjunction with the external evaluation of the 2 nd Cycle of the National Program for Improving Access and Quality (PMAQ) from

\section{Introdução}

Este artigo é resultado de um trabalho de conclusão do Curso de Especialização em Avaliação de Serviços em Saúde com ênfase em Educação Permanente da Universidade Federal de Ciências da Saúde de Porto Alegre em parceria com o Programa de Melhoria do Acesso e da Qualidade (PMAQ) do Ministério da Saúde. Este curso teve como objetivo realizar uma formação interprofissional de trabalhadores e trabalhadoras do campo da saúde acerca da eficiência, eficácia e
January 2014 in the state of Pará. Tutors of the specialization also coordinated teams of professionals who collected data on the state's family health teams. Moments were created to discuss aspects arising from the evaluation process and the strangeness caused by daily work. The organization of the work was shared and discussed by the teams. The specialization course was rich in exchanges of experiences with students from several states, but with few spaces of active participation of students in the construction of the course. A culture of health service evaluation is being germinated by PMAQ. Stimulated behavioral changes can deliver better health outcomes for the population. In this way, the experience of evaluation needs to be understood as a social and political transformation process.

Keywords: Permanent Education; Health Evaluation; Primary Health.

efetividade das estruturas, processos de trabalho e resultados relacionados ao risco, acesso e satisfação dos cidadãos frente aos serviços do Sistema Único de Saúde (SUS) na busca de maior resolubilidade e qualidade. Assim, pretende-se contar aqui a vivência de tutoria da autora principal nesta especialização, discutindo a Educação Permanente e sua relação com a Avaliação de Serviços de Saúde.

O início da avaliação externa do 2ㅇ Ciclo do 
PMAQ aconteceu a partir de janeiro de 2014 no estado do Pará. Houve uma aula inaugural onde foi possível ter o primeiro contato com a proposta do curso de Especialização e a alegria de conhecer os alunos que estariam sob a tutoria, além dos avaliadores que comporiam a equipe de campo.

Envolvida com a coordenação do trabalho de duas equipes de avaliação Externa do Programa Nacional de Melhoria do Acesso e da Qualidade da Atenção Básica (PMAQ-AB), uma gama de possibilidades se acendeu para mim. A associação do trabalho a um curso de Educação à Distância voltado para avaliação de serviços de saúde foi um insight muito inteligente. Além de aproximar os avaliadores e supervisores e produzir conhecimento coletivo sobre o trabalho, era uma oportunidade para que muitos pudessem ganhar o reconhecimento de um diploma de especialização, este título embora aparentemente simbólico, estava presente na fala de muitos como uma conquista importante.

O instrumento de avaliação se caracterizava, basicamente, por uma ferramenta dura, com muitas questões de base quantitativas, fechadas e com pouca abertura para outros apontamentos e observações do profissional entrevistado. Desse modo, incentivei a equipe a produzir um diário de campo consistente, onde pudessem livremente inserir todas as impressões sobre o trabalho, a viagem, a equipe, a unidade, dificuldades, acertos, apostas, surpresas, enfim, uma ferramenta aberta à construção. 0 importante também era olhar de forma atenta para o seu trabalho e ter sempre a consciência de que nenhum instrumento vai dar conta da realidade, da potência viva do cotidiano das equipes de saúde da família e tudo que pudessem registrar nos diários era mais uma forma de conhecimento.

A estrutura do curso foi adaptada a partir do conteúdo de outros cursos, como a Especialização ligada ao Programa de Valorização do Profissional da Atenção Básica (PROVAB). Desse modo, alguns módulos exigiam uma vivência específica do aluno em unidades de saúde, o que não se enquadrava ao perfil de muitos. Outro aspecto a ser considerado era que grande parte do curso seguiu concomitantemente com o trabalho de campo, associado ao cronograma apertado, ao peso de algumas atividades e a dificuldade de conexão em alguns municípios do interior do Estado, contribuiu para desestimular os alunos em continuar realizando os módulos. Muitas vezes somos capturados pelos instrumentos, pelas disciplinas duras e pela própria estrutura 
do curso, mas aos poucos fui tentando produzir furos e fendas para, assim, poder estabelecer outros modos de produção de conhecimento, nem sempre com resultados esperados.

Pretendi, de forma concisa, pontuar os aspectos marcantes e estruturantes da minha trajetória profissional. A formação e o desenvolvimento das minhas atividades profissionais sempre caminharam em direção a movimentos de reflexão e prática. $O$ interesse pela atividade docente segue essa lógica, na medida em que permite a troca de experiências e conhecimentos entre pessoas, promove crítica e criação de novas ideias. Acredito que a prática de ensino e pesquisa é um ganho para o aluno, mas igualmente para o docente, e que as experiências relatadas aqui puderam contribuir de maneira importante para esta tutoria.

\section{A Educação Permanente em mim}

A educação permanente em saúde ganha estatuto de política governamental e é palco de debate entre vários autores na atualidade ${ }^{1-5}$. Ceccim e Feuerwerker ${ }^{5}$ fazem uma análise crítica das nossas práticas de formação em saúde e desenvolvem o conceito de quadrilátero da formação, constituído pelos aspectos de ensino, gestão, atenção e controle social. A partir da constatação de que "a formação dos profissionais de saúde tem permanecido alheia à organização da gestão setorial e ao debate crítico sobre os sistemas de estruturação do cuidado, mostrando-se absolutamente impermeável ao controle social sobre o setor" ${ }^{5: 42}$, se propõem a repensar os modelos de educação no país.

A educação permanente, nessa perspectiva, se mostra como uma ação transdisciplinar e descentralizadora onde é preciso "implementar espaços de discussão, análise e reflexão prática no cotidiano do trabalho e dos referenciais que orientam essas práticas [...] ativadores de processos de mudança institucional e facilitadores de coletivos organizados para a produção" ${ }^{2}$. Trata-se de uma aposta no protagonismo dos trabalhadores, em debate com os usuários, gestores e os processos de ensino e aprendizagem.

O tempo da produtividade, do fazer mais em menos tempo, é muito diferente do tempo da saúde. Dessa maneira, a inserção de pessoas nessa lógica se restringe e, cada vez mais, os que trabalham são intimados a abrir mão do prazer, do desejo e daquilo que dá sentido ao fazer. De acordo com Guattari, "A única finalidade aceitável das atividades humanas é a produção de uma subjetividade que enriqueça de modo contínuo sua relação com o mundo" $2: 33$ 
Um autor que contribui para compreensão das tecnologias de trabalho e as discussões entre trabalho e saúde, Merhy ${ }^{3,7}$ inclui no hall das tecnologias uma diferenciação entre os conceitos de tecnologias "duras", tecnologias "leves" e tecnologias "leve-duras" ampliando esta definição e afirmando a valorização da dimensão relacional dos atos de saúde, ou seja, aquilo que é da ordem do humano e, portanto, do encontro entre seres humanos. As tecnologias duras são os equipamentos, máquinas e normas necessárias ao trabalho da saúde, as leves são aquelas que permitem operar os processos relacionais do encontro entre o trabalhador da saúde e o usuário (acolhimento, vínculo, gestão como uma forma de governar processos de trabalho) e as leveduras são os saberes estruturados que operam os processos de trabalho em saúde (em particular a clínica e a epidemiologia).

Desse modo, para abordar a temática do trabalho é fundamental tomar como referência o conceito de tecnologia como um conjunto de saberes e ações aplicadas à produção de algo que o trabalhador utiliza para dar conta do ato complexo de cuidar. O modo tecnológico de produção da saúde pode passar por diversos dispositivos de mudança no jeito de fazer saúde, sem, entretanto, mudar seu núcleo tecnológico, ou seja, a transformação precisa ser profunda a ponto de alterar a lógica da produção. Dito de outra forma, para que se modifique a lógica "procedimento centrada" ou "médico centrada", é preciso alterar a hegemonia do trabalho morto, para uma nova concepção, baseada no trabalho vivo em ato e nas necessidades dos usuários ${ }^{7}$.

Merhy ${ }^{7}$ é também um dos principais autores brasileiros a contribuir para a compreensão das transformações e da especificidade do trabalho em saúde, lançando mão de conceitos fundamentais para pensar a singularidade da atividade em saúde, que são os de "trabalho morto" e "trabalho vivo em ato". Existem tipos de trabalhos onde o que predomina são as relações com o trabalho morto e outros em que o trabalho vivo prevalece. Para o autor, os atos de cuidar em saúde são trabalhos vivos centrados. O trabalho vivo, portanto, opera demarcando territórios, interesses e constrói um certo mundo para si. Nas palavras de Merhy ${ }^{7: 48}$,

O trabalho em saúde é centrado no trabalho vivo em ato permanente [...] atua distintamente de outros processos produtivos nos quais o trabalho vivo em ato pode e deve ser enquadrado e capturado globalmente pelo trabalho morto e pelo modo de produção [...] operando como 
tecnologias de relações, de encontros de subjetividades, para além dos saberes tecnológicos estruturados, comportando um grau de liberdade significativo na escolha do modo de fazer essa produção.

Desse modo, durante todo o trabalho de supervisão do $\mathrm{PMAQ}$, procurei passar à minha equipe de avaliadores a importância do trabalho que estavam desenvolvendo, empoderando-os enquanto grupo para que pudessem refletir sobre a prática deles e se percebessem capazes de contribuir para a melhoria da qualidade dos serviços prestados para a população do seu estado. Ao conhecer a realidade da saúde pública no seu estado, também puderam se indignar com determinadas situações e se orgulhar com outras. Essa experiência propicia exercício de cidadania e controle social, ao pensar sobre a situação em as pessoas que vivem e se colocar no lugar de quem pode fazer ou que já está fazendo alguma coisa.

Sempre que possível, criávamos espaços para discutir aspectos decorrentes do processo de avaliação e estranhamentos provocados pelo cotidiano de trabalho. Essa troca de experiências foi importante para todos e sem perceber estava contribuindo para o nosso processo de formação. Desde o início, sempre procurei me colocar disponível em tempo integral, mas ainda, fortalecia neles o papel gestores e planejadores do seu trabalho. A organização do trabalho era, desse modo, compartilhada e discutida pela equipe toda. Sempre que algum imprevisto ocorria, escutava cada um dos envolvidos e tomávamos a decisão juntos. Mas, se por acaso, não tivesse tempo hábil de ser acionada, eu os incentivava a decidir coletivamente com os colegas de equipe, sem deixar de considerar os aspectos éticos e profissionais inerentes ao trabalho que estavam desempenhando.

O diário de campo foi um instrumento potente para reflexão e registro das principais impressões, decisões, acontecimentos diversos ocorridos no desenvolvimento do trabalho. Nesse dispositivo era possível rever o dia-a-dia de trabalho, repensar estratégias que não deram certo, aprender com os erros, sentir-se recompensado pelos acertos, entre outros. 0 uso dessa ferramenta foi incentivado com insistência e constitui hoje um material rico também para análise e produção de conhecimento.

Em relação ao Curso de Especialização, vou falar a partir do lugar de aluna e de tutora. Como aluna, foi muito bom poder trocar experiências com alunos de outros estados, com vivências 
diversas e realidades distintas. Conhecer um pouco de cada parte e saber o que as pessoas estão pensando por aí a fora, esta foi, de fato, uma experiência particular. Em termos de conteúdo, acho que o módulo de educação permanente poderia ter sido mais trabalhado e ter sido transversal ao curso como um todo. Encontrei poucos espaços em que pudéssemos participar mais ativamente da construção do curso, bem como exercícios de reflexão coletiva, voltados para problematização das dificuldades encontradas no cotidiano de trabalho das equipes envolvidas com a Avaliação Externa do PMAQ.

Já como tutora, procurei incentivar as participações e trocas entre os alunos, dando suporte as dúvidas de maneira presente e fazendo avaliações dialogadas. Fizemos quatro encontros presenciais com os alunos do Pará para tentar minimizar as eventuais dificuldades com conteúdo ou com as ferramentas da plataforma, discutir impressões, debater assuntos dos módulos do curso e criar uma aproximação entre tutores e especializandos. Do mesmo modo, preciso dizer que senti falta de um apoio pedagógico da coordenação geral do curso de forma mais presente. No decorrer do mesmo, tivemos algumas reuniões com a coordenação pedagógica local e entre os encaminhamentos feitos, recebemos pouco retorno ou este não ocorria em tempo hábil. Mas de forma alguma esses atravessamentos prejudicaram o trabalho ou desmotivaram a valiosa convivência entre os alunos ao longo dos conteúdos desenvolvidos.

O PMAQ como uma ferramenta de monitoramento e avaliação, permite melhorar a gestão de processos e a organização dos serviços, do mesmo modo, pode ampliar a negociação e o diálogo entre os gestores, as equipes e os usuários. A partir da problematização dos processos e das práticas de cuidado - do debate envolvendo todos os atores, suas necessidades e interesses em disputa - caminharemos na direção da produção de ações e serviços de saúde com qualidade e erguemos o último vértice desse quadrilátero, que diz respeito à construção conhecimento, de modo coletivo e democrático.

\section{O que pode a avaliação?}

O campo da avaliação em saúde no Brasil passa por um momento de crescimento, com a pluralidade de objetos e de abordagens metodológicas que abriga, se constituindo em importante instrumento de produção de conhecimento. Novaes ${ }^{8}$ aponta algumas dificuldades na identificação e sistematização 
da informação produzida na área e se fundamenta em uma ampla revisão da literatura produzida nos anos 90 , principalmente em língua inglesa, para construir tipologias de análise sobre avaliação em saúde.

Os processos de avaliação devem ter por objetivo produzir conhecimento, subsidiar a tomada de decisão, a formulação de políticas para o setor, além de estabelecer mecanismos de regulação e melhoria das práticas nos serviços de saúde. Admitindo a impossibilidade de se realizar uma avaliação isenta ou neutra e recusando o estabelecimento de classificações simplistas, Novaes ${ }^{8}$ não busca identificar avaliações melhores e mais adequadas, mas sim analisar quais os principais condicionantes que incidem em tais avaliações.

A autora aponta três grandes tipologias dos processos avaliativos: a pesquisa de avaliação (avaliação aleatória) que tem como principal objetivo a produção do conhecimento; a avaliação para decisão e a avaliação para a gestão. Desse modo, o conhecimento aparece como recurso de poder na medida em que a investigação adquire papel específico, se desenvolve a partir de escolhas metodológicas mais apropriadas de acordo com cada objetivo, possibilita intervenções e tomada de decisões "bem informadas".
Um mesmo objeto pode ser avaliado nas suas diversas dimensões, no caso da saúde, podemos analisar o cuidado individual, mas também os seus níveis mais complexos de intervenção e de organização, como serviços, políticas de saúde, programas específicos ou até sistemas de forma mais global. Há uma enorme diversidade de formas, metodologias, abordagens e enfoques teóricos acerca dos processos de avaliação. "Sob o rótulo de 'avaliação' reúnem-se diversas atividades, cujo aspecto varia desde um julgamento subjetivo do desenvolvimento de determinada prática social” $^{\text {9:80 }}$.

Entre as avaliações em saúde, Novaes ${ }^{8}$ destaca três grupos: a avaliação tecnológica em saúde, avaliação de programas em saúde e avaliação, gestão e qualidade em saúde. Há uma articulação entre a produção de conhecimento e a sua utilização prática e política. Daí a importância da realização de pesquisas em serviços de saúde, como um recurso efetivo para as mudanças pretendidas, levando em consideração seus limites e potencialidades.

Donabedian ${ }^{10}$ desenvolveu uma discussão sistemática sobre a questão da avaliação. Criou um modelo para o entendimento da avaliação de qualidade em saúde, a partir dos conceitos de Estrutura, Processo e Resultado. Estrutura se refere basicamente aos recursos físicos, 
humanos, materiais e financeiros necessários para a assistência em saúde. O Processo diz respeito às atividades que envolvem profissionais de saúde e pacientes, seu trabalho e suas relações. Já os Resultados se caracterizam ao produto final da assistência prestada, considerando saúde, satisfação de padrões e de expectativas. Desse modo, quando os processos avaliativos envolvem estas três dimensões, mais abrangente será a análise e abordagem da complexidade necessária às boas práticas em saúde.

\section{É fundamental reconhecer o contexto, o} momento histórico e político em que foram geradas as avaliações para que em uma nova avaliação, os resultados sejam reinterpretados e, caso necessário, sejam feitas adaptações. Além do contexto, a quantidade e qualificação de recursos humanos, a informação e os recursos materiais disponíveis são pontos essenciais que não deverão ficar de fora da análise.

De acordo com Silva e Formigli", "na prática cotidiana dos serviços de saúde no Brasil, a avaliação não é feita rotineiramente ou, pelo menos, enfrenta dificuldades metodológicas e operacionais não respondidas completamente no plano da investigação". Ainda insuficientes, produzem poucas intervenções capazes de modificar uma dada situação de saúde.
O Programa Nacional de Melhoria do Acesso e da Qualidade da Atenção Básica (PMAQ), lançado em 2011, é uma ferramenta de avaliação de serviços e ações de saúde que tem como objetivo promover a melhoria do acesso e da qualidade da atenção à saúde. É uma aposta que o Ministério da Saúde faz na tentativa de monitorar os serviços básicos de saúde e qualificar as equipes de atenção primária. Acredito que o PMAQ está ajudando a construir uma cultura de avaliação de serviços, bem como a impulsionar mudanças de comportamento que viabilizem melhores resultados na saúde da população, tomando a experiência de avaliação como processo social e político de transformação.

O Curso de Especialização em Avaliação de Serviços contribuiu para a construção de um pensamento crítico sobre avaliação em saúde, considerando a complexidade da área e alertando para o cuidado de produzirmos um conhecimento mais fundamentado cientificamente.

\section{Considerações Finais}

Construir um processo de implementação da Educação Permanente em Saúde para o SUS é um desafio tão grande como a tarefa de instituir a avaliação dos serviços visando a melhoria da prestação das ações em saúde para a 
população. Por outro lado, muitas experiências de educação permanente e novos processos de formação vêm acontecendo, sem que se tenha designado formalmente esse lugar como espaço de formação ou capacitação do trabalhador para o exercício das suas funções.

\section{Desse modo, é necessário reconhecer e} valorizar os encontros que se tecem no cotidiano das práticas em saúde, onde se está permanentemente produzindo conhecimento e, trazer a perspectiva da avaliação como um instrumento para a tomada de decisão mais informada e ferramenta fundamental para, não apenas a gestão macro da organização dos sistemas e serviços de saúde, mas também para a gestão micro dos processos de trabalho, reflexões e transformação de práticas voltadas para a qualidade do cuidado em saúde.

O recorte que o tempo deste trabalho impõe, faz com que germinem questões ainda latentes, perguntas até então não formuladas, achados ainda por descobrir e perspectivas que se apresentam para futuras investigações. Esperamos que este texto sirva como material para que outros estudantes, pesquisadores, gestores e trabalhadores possam refletir seus objetos de análise e de prática, bem como construir outras questões e produções de conhecimento para área.

\section{Referências}

${ }^{1}$ Ministério da Saúde. Secretaria de Gestão do Trabalho e da Educação na Saúde. Política Nacional de Educação Permanente em Saúde. Brasília: Ministério da Saúde; 2009.

${ }^{2}$ Ceccim RB. Educação Permanente em saúde: desafio ambicioso e necessário. Interface - comunicação, saúde, educação. 2005;9(16):161-177.

${ }^{3}$ Merhy EE. O desafio que a educação permanente tem em si: a pedagogia da implicação. Interface - comunicação, saúde, educação. 2005;9(16):161-177.

${ }^{4}$ Rovere M. Comentários estimulados por la leitura del artículo "educação permanente em saúde: desafio ambicioso e necessário". Interface - comunicação, saúde, educação. 2005;9(16):161-177.

${ }^{5}$ Ceccim RB, Feuerwerker LCM. O Quadrilátero da Formação para a Área da Saúde: Ensino, Gestão, Atenção e Controle Social. Physis: Revista de Saúde Coletiva. 2004; 14(1): 41- 65.

${ }^{6}$ Guatarri F. Caosmose: um novo paradigma estético. São Paulo: Editora 34; 1992.

${ }^{7}$ Merhy, EE. Saúde: Cartografia do trabalho vivo. São Paulo: Hucitec; 2007.

${ }^{8}$ Novaes, HMD. Avaliação de programas, serviços e tecnologias em saúde. Ciência e Saúde Coletiva. Revista de Saúde Pública. 2000;34(5):547-59.

${ }^{9}$ Silvia, LMV, Formigli VLA. Avaliação em Saúde: Limites e Perspectivas. Cadernos de Saúde Pública. 1994;10(1):80-91

${ }^{10}$ Donabedian, A. Basic approaches to assessment: structure, process and outcome. The definition of quality and approaches to its assessment: explorations in quality assessment and monitoring. 1980; 1:77-128. 